\title{
TNF Repeat
}

National Cancer Institute

\section{Source}

National Cancer Institute. TNFRepeat. NCI Thesaurus. Code C129764.

A group of five microsatellite repeats that are either upstream of the LTA gene or downstream of the TNF gene. TNF repeat polymorphism may influence either lymphotoxin-alpha or tumor necrosis factor protein expression, may be associated with certain diseases, or may be used to facilitate population genetics studies. 